educational materials on ACP and exclusion of ACP in clinical notes. After multiple interventions over 6 months, mean monthly completed ACP documentation rose from baseline of $5.5 \%$ to $28.8 \%$, with highest achievable completion of ACP documentation at $50 \%$. Though the target of completed ACP documentation was not met, there was an increase in the number of ACP discussions and documentation conducted by the department with increase awareness. This promotes a community of practice where ACP is offered readily.

Conclusion Changes in work processes and incorporation of ACP as part of clinical service delivery can improve ACP engagement in patients with dementia and their caregivers.

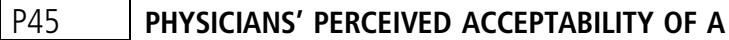 QUESTION PROMPT LIST FOR DEMENTIA AT THE END OF LIFE}

${ }^{1} \mathrm{~S}$ Heck*, ${ }^{2} \mathrm{JT}$ Van der Steen, ${ }^{3} \mathrm{CM}$ Juffermans, ${ }^{3} \mathrm{YM}$ Van der Linden, ${ }^{3} \mathrm{MM}$ Garvelink, ${ }^{3}$ WP Achterberg, ${ }^{3}$ RTCM Koopmans. 'Leiden University, Leiden, Netherlands; ${ }^{2}$ Leiden University Medical Centre, Leiden, Netherlands

\subsection{6/spcare-2019-ACPICONGRESSABS.128}

Background Question prompt lists can support communication between patients, relatives and professional caregivers. Examples of questions encourage to ask about topics that are relevant to patients and relatives, which may, for example, enhance participation in decision making and improve outcome. We developed a question prompt list about end of life for people with dementia and their relatives. Physicians' endorsement of its use and perceived acceptability is essential for effective implementation.

Methods In 2018, we surveyed 65 physicians (elderly care physicians and general practitioners) to evaluate the list. The survey was based on decision aid evaluation methodology including a validated acceptability scale ranging 15-75 with scores of 45 and meaning the aid is acceptable. Subsequently, we interviewed those physicians with either very high or very low acceptability scores.

Results The mean acceptability score was 51 , but the physicians differed substantially in their evaluations of acceptability (SD 11). One third (32\%) felt unable to answer the questions in the question prompt list. The interviews indicated this was bothering for some, and we explored this using the quantitative data. With inability to answer all questions, acceptability was lower and more variable (46 SD 13 vs. 54 SD 8).

Conclusions When accompanied by physician guidance and training, the question prompt list may fill a gap in seeking ways to develop advance care planning specific to dementia. An improved version of the question prompt list will be tested as part of two innovative advance care planning interventions.

\section{P46 IMPROVING THE QUALITY OF PREFERRED PLAN OF CARE DISCUSSION USING A COMPANION GUIDE FOR PATIENTS WITH ADVANCED DEMENTIA}

E Ho*. Tan Tock Seng Hospital, Singapore, Singapore

10.1136/spcare-2019-ACPICONGRESSABS.129

Background For patients with advanced dementia, preferred plan of care conversations helps to improve end of life outcomes by creating an opportunity for their carers to discuss about the circumstances in which they would prefer to limit life prolonging treatments and instead opt for an approach that prioritizes quality of life.

A discussion aid provides patients and families with structured information about clinical choices and help enhance the clinical decision making.

This will improve time efficiency and quality of informed decision making by increasing disease specific knowledge, reducing decisional conflict and promote evidence-based treatments.

Objectives Use of a discussion guide to improve the quality of discussion for patients with advanced dementia.

Methodology This study was a cross sectional study conducted in August 2018. The target population are physicians in the department of Geriatric Medicine, Tan Tock Seng Hospital. A discussion aid was designed to present balances, evidence-based information about risk, benefits and alternatives of clinical decisions to aid in discussion of end of life care in patients with advanced dementia.

Results Of the 43 physicians surveyed, 30\% of them strongly agreed; 62\% agreed; 4\% Neither agree nor disagree and 2\% disagree that the discussion aid will improve quality of ACP discussions.

Conclusion Most of the physicians surveyed felt that the discussion guide will improve the quality of communication and ACP discussion in patients with advanced dementia. They are also likely to use this guide for future ACP discussions.

\section{P47 IMPACT OF THE MENTAL CAPACITY ACT ON PLACE OF DEATH IN HEART FAILURE PATIENTS WITH OR WITHOUT COMORBID DEMENTIA}

W Gao, JM Beattie*, T McDonagh, IJ Higginson. King's College London, London, UK

\subsection{6/spcare-2019-ACPICONGRESSABS.130}

Background The Mental Capacity Act (MCA) came into force in England in 2007 to foster surrogate decision making, considered particularly relevant to advance care planning for those at the end of life. This study examined the impact of the MCA on the place of death(PoD) for heart failure(HF) patients sometimes affected by the loss of capacity.

Methods A national population-based retrospective cohort study of death registry data(DRD) in England, 2001-2016. All patients dying from HF (ICD-10: I50) were included. Capacity status designation was based on documentation of dementia (ICD-10: G30, F01, F03) as a comorbidity on DRD. Using binomial regression analysis, we compared PoD of HF decedents with and without comorbid dementia before and after MCA enactment.

Results 113,773 HF patients were included of whom 7,473 $(6.5 \%)$ had dementia, these tending to be older, female, and with more comorbidities. $66 \%$ and $19 \%$ of HF patients with capacity died in the hospital or care home, respectively, the two commonest PoDs; in contrast, corresponding figures for those without capacity were $49 \%$ and $43 \%$. Hospice as a PoD was rare $(\leq 0.5 \%)$ in both groups. Over the above period encompassing MCA implementation, PoD for $\mathrm{HF}$ patients with capacity shifted from hospital to home or care home (adjusted prevalence ratio (APR): 1.024[95\% CI: 1.0211.026]; $\mathrm{p}<0.001)$; this trend less evident in those lacking capacity (APR: 0.995[0.981- 1.010]; $\mathrm{p}=0.51$ ). 